\title{
A Hybrid Approach to Increase the Scalability in VANETs
}

\author{
Kavita $^{1}$,Neera Batra ${ }^{2}$ and Rishi Pal Singh ${ }^{3}$ \\ ${ }^{1}$ Ph.D. Scholar, CSEDeptt., MMU Mullana, Ambala, India. \\ ${ }^{2}$ Associate Professor, CSEDeptt., MMU Mullana, Ambala, India. \\ ${ }^{3}$ Associate Professor, CSE Deptt., G.J.U. S. \& T., Hisar, India.
}

\begin{abstract}
Scalability in VANET environment is an important issue of concern in recent years. Many approaches have been presented in the recent years and compared their merits and demerits with the existing approaches. In the present work a hybrid technique extension to the GPSR protocol is presented which is based on the content based filtering approach and the clustering approach. The filtering approach uses Bloom Filter approach while the clustering uses the mobility based approach. The results are compared with the GPSR approach and the mobility based single hop network approach on the basis of different performance parameters. The results are also compared with the scalable clustering based approach which verifies the validity of the algorithm by varying the number of vehicles in the network. The Scalability is increased in the network with the improved performance and the stability of the network on the basis of performance parameters like cluster head and cluster member's lifetime. Results also verifies that the present approach is better as compared to the basic approach.
\end{abstract}

Keywords:Scalability, VANET.

\section{INTRODUCTION}

In wireless network, various routing protocol had been implemented. But in recent years GPSR routing protocol is mainly popular as compared to other traditional existing protocols [1].GPSR is routing protocol which is used for wireless datagram network and it uses the location of routers to make packet forwarding decisions. This routing protocol makes greedy forwarding decision by using the information about immediate neighbor of routers in the network.Scalability is playing a vital role in the implementation of routing protocol in wireless network. The term scalability means that the capability to maintain network development to integrate more nodes that might not be predictable during the preliminary network design stage [2].Wireless sensor network should maintain the scalability of network accordingly to the routing protocol used. Over the last few years, vehicular ad-hoc network have turned into a significant research area. In vehicular ad-hoc networks, the problem of scalability is arises in various contexts [3]. In the distributed networks, scalability is very vital characteristics. Scalability is simply defined as the capability to deal with the nodes without getting loss in performance or enhance in administrative complexity. Moreover, design of protocol has great impact on scalability. Scalability used in WSN, GPSR is a major challenge because of its increasing node density and increasing node numbers [4]. Routing protocol is said to be good protocol only when if it is adaptive and scalable to the variation in network topology. Greedy Perimeter Stateless Routing (GPSR) is basically routing protocol depending on the geographical position that is appropriate for vehicular adhoc network (VANET). This GPSR routing protocol is proposed by Brad Kard and H.T Kung in 2000. In this routing protocol, every node transmits its location information periodically to the neighboring nodes [5]. The information received by neighboring nodes is stored in the neighboring table. The forwarding node selects the next hop forwarding node according to the both neighbor location information and destination location. In order to promote the desired packet effectively, GPSR utilizes the information of nearest neighbor of destination.

In VANET using GPSR, every vehicle determine the number of its neighbor vehicles and their averages speeds and then transmit this information to other vehicles in order to prevent them approaching the busy location. Moreover in some cases, the information can be communicated by those vehicles which are moving in the other direction so that it may be communicated faster to the vehicles toward the congestion location. Information like weather, road surface, construction zones, highway rail intersection, and emergency vehicle signal is also collected by vehicles. One very significant requirement for the unbeaten and also sustainable deployment of VANETs is the scalability of the applied information broadcasting method [9]. The separately acting networks have to make sure delivery of data to those nodes which are interested in it with low latency.

VANET (Vehicular Ad hoc networks) is a type of Mobile Ad hoc Network (MANET). Every node is acting as router to transfer information from one node to another node. In VANET, there are two type of communication. One is vehicle to vehicle $(\mathrm{V} 2 \mathrm{~V})$ and the other is vehicle to fixed road side equipment (V2R) [11]. In both the methods vehicles can communicate to other vehicles or road side unit either directly or through multiple hops. $\mathrm{V} 2 \mathrm{~V}$ communication is the communication between direct vehicle to vehicle [16]. Vehicular ad hoc wireless networks (VANETs) are popular among various researchers in the field of wireless networking as well as automotive industries.Vehicular ad-hoc networks are those networks that give the informationamong adjoining vehicles or nearby fixed equipment. Each vehicle records and provides the information such as place, speed or route that information from other vehicles in network. Congestion detection is one of the multiple applications of VANETs.The Vehicular Ad-hoc Network has been presented in various areas, such as detecting nearest collisions and giving warning signals to aware driver. Since VANET has ability to provides 
a variety of services. These services are provided by VANET often based on association or among vehicles which are furnished with a relatively motion sensors and GPS units [20]. Awareness of the specific location is vibrant to the every vehicle in VANET so it can provide an accurate data to aristocrats.VANETs have some unique characteristics like Vehicles move at high speed. Mobility patterns are somehow predictable as movement is constrained by road infrastructure. In some situations such as highway traffic, the mobility patterns become highly predictable. These VANET networks have large coverage area. Vehicles travel over long distances and traffic information may be useful to vehicles hundreds of miles away. In these networks, vehicles have a high cost and therefore can be equipped with additional sensors without significantly impacting the total cost. Vehicles travel long distances in a small amount of time when compared to other mobile networks. This is extremely dynamic as vehicles go in and out transmission range quite rapidly. Power consumption is not a major concern in VANET networks.

The paper is organized as follows. In section 2 related work is presented. In section 3, methodology is discussed which is used in this paper. The result is presented in section 4. And finally conclusion and future scope is presented is section 5 .

\section{RELATED WORK}

In the work presented in [1], proposed Scalability analysis for WSN routing protocols. The proposed protocol is used to reducethe routing overhead and delay. Concurrently it is used to increase the reliability of ad-hoc network. Moreover, comparison of proposed approach with existing approach has been presented in this paper and it indicates that the proposed approach attains better performance than those of existing methods. Another effort is presented based on Location-based Routing protocol by Shen et al in [2]. The proposed routing protocol distributes the network in to different regions and arbitrarily chose the nodes in region as intermediate relay nodes. Moreover, it hides the information between the receiver to make stronger source and destination protection. The result shows that the proposed protocol is very efficient and can secure source, route and destination. A scalable routing protocol for ad-hoc network is relied on the geographical location management scheme [3]. The result concluded that the proposed protocol is better for large networks where nodes are travel at very high speed.Mikki et al [4] proposed a location aided routing protocol for wireless networks. The proposed protocol is very efficient and used for mobile networks. In the proposed protocol, wireless protocol is used and it is divided into six equal sub areas. NS-2 simulator is used for simulation and the result indicates that the proposed protocol makes an improvement in the control packet overhead and delivery ratio. A novel position-based routing protocol is proposed in [5].in the given approach, packets are routed from one junction to another.The direction and position of next hops are pre-considered with different ratios depending on the estimated density of the path.An approach has been proposed in [6] which are used to minimize the routing overhead and delay. Simultaneously it is used to enhance the reliability of ad-hoc network that utilizes LAR protocol. The proposed approach is based on the two-hop neighbor information and forwarding mechanism. Moreover, comparison of proposed approach with existing approach has been presented in this paper and it indicates that the proposed approach attains better performance than those of existing methods [6]. A routing strategy for vehicular ad hoc networks in city environments has been presented in [7] .In this paper, geographic source routing' (GSR) has been proposed, which combines position-based routing with top logical knowledge, as a promising routing strategy for vehicular ad hoc networks in city environments [7].In (8), scalable content oriented routing has been discussed. In this paper, scalable proactive scheme has been proposed in order to tackle the challenges of VANET's. In the simulation result, proposed approach shows high content availability with low latency rate. Moreover proposed approach improves its response time by $45 \%$ as compared to other existing system and it reduces the traffic by $85 \%$. In this paper given in [9], hybrid approach has been proposed for privacy preserving authentication. The proposed approach is combination of pseudonym-based approaches and the group signature-based approaches. The given approach uses the efficient and light-weight pseudonyms and it is use for message authentication as well as a trapdoor in order to provide conditional anonymity. The result demonstrates that the feasibility of given proposed approach in terms of end-toend delay and packet delivery ratio. This paper [10] investigates the information delivery delay for the rationale of roadside unit (RSU) deployment in a VANET with intermittent connectivity. The correctness and accuracy of the derived mathematical model is verified and the impacts of different parameters on the average information delivery delay are investigated through simulation results.

This paper proposed that wireless sensor network has vital applications like as remote environmental target tracking and monitoring. This has enabled by availability, specifically in later years, of sensors which are cheaper, smaller, and intelligent. Author proposed an Adaptive Disjoint Path Vector (ADPV) algorithmin [12]. The main purpose of this proposed algorithm is to provide security to super nodes and it is attain its aim by adjusting the transmission power. The simulation result demonstrated that the proposed algorithm can attain around $95 \%$ of its objective and it is better algorithm for securing super nodes. In this paper, Mishra,et al. [13] proposed the energy efficiency which is a key issue to design the protocol, because its sensor nodes have time energy of battery backup. There are multiple modern protocols that extend the lifetime of wireless sensor network with efficient battery power of sensor node. In this proposed a newest protocol Energy Level Stable Election Protocol in the Wireless Sensor Network.Mansouri. V. S et al.[14] proposed a simple transport protocol for wireless sensor Networks. The basic problems of wireless sensor networks are reliability, battery depletion and fading. Authors have tackled reliability and battery depletion by path redundancy and fading by using diverse combination of independently fading signal paths. Gradient broadcast algorithm is used as routing protocol for simulation.

\section{PROPOSED METHODOLOGY}

The scalability issue of the network in Vehicular Ad-hoc 
Network architecture is addressed using the hybrid technique. The hybrid technique is the combination of the content based technique and the clustering technique.

\section{Content based Message Filtering Technique}

In this technique the message content is filtered and only the important information regarding the controlling of the vehicle is propagated to the road side unit while discarding the advertisement message information. The filtering algorithm used in the hybrid technique is based on the point to point content discovery structure. The Probabilistic data structure is used to test whether an input is element of the super set. The super set is a set of advertisement messages and can be directly discarded is found the member of the set. The existence of the element is determined by searching the elements of the array. False positives as an output of the search can be achieved but false negatives are not possible. The probability of false positives in an $\mathrm{m}$ bit array with and $\mathrm{k}$ number of elements, $n$ be the number of items searched is:

$$
\left(1-e^{-\frac{k n}{m}}\right)^{k}
$$

\section{Clustering Technique}

Clustering of vehicles in the VANET is an efficient approach for improving the scalability of the network. A matrix is created with the vehicle location, direction, velocity and acceleration. Firstly, with the help of these parameters relative velocity, relative distance between the vehicles is calculated. Two vehicles $V_{i}$ and $V_{j}$ are initially selected which are within range of each other. Now the relative distance, relative velocity, relative acceleration and direction of each vehicle form the selected vehicles is calculated. The vehicles having the same direction, distance within the considered range and same acceleration are considered. The vehicles having the minimum distance with the two vehicles is considered to be in the cluster of the vehicle.

\section{Algorithm}

\section{1: Start}

2: initialize $n \leftarrow$ location, where $n$ is the node

3: $\quad$ for $\boldsymbol{i}=\mathbf{0}: \boldsymbol{N}$, where $N$ is the total no. of nodes

4: $\quad R_{i} \leftarrow$ Range $\left(n_{i}\right)$, Range of $i^{\text {th }}$ node

5: $\quad N u_{1}, N u_{2}$, Consider two random nodes in range with one another

6: $\quad \boldsymbol{C a l c V} \leftarrow \operatorname{Velocity}\left(n_{i}, N u_{1}, N u_{2}\right)$, Relative Velocity between vehicles

7: $\quad \boldsymbol{C a l c} \boldsymbol{A} \leftarrow \operatorname{Acc}\left(n_{i}, N u_{1}, N u_{2}\right)$, relative Acceleration

8: $\quad \boldsymbol{C a l c D} \leftarrow$ Distance $\left(n_{i}, N u_{1}, N u_{2}\right)$, Distance between vehicles and other neighboring nodes

9: $\quad$ if $\left(\right.$ Calc $\left._{i}, N u_{1}>\operatorname{CalcD}_{i}, N u_{2}\right)$

10: $\quad$ if $\left(\operatorname{dir} N u_{2}=\right.$

$n_{i}, C$ alcV, Calc $\left.A\right)$, where relative velocity and acceleration is considered as positive

11:

$\operatorname{Cluster}\left(\mathrm{Nu}_{2}\right) \leftarrow \mathrm{CalcD}_{i}$

12:

else

13: $\quad$ if $\left(\operatorname{dir} N u_{1}=\right.$

$n_{i}, C$ alcV, CalcA), where relative velocity and acceleration is considered as positive

14: $\quad$ Cluster $\left(N u_{1}\right) \leftarrow$ Calc $_{i}$

15: foreach Cluster: $C$

16: $\quad$ while $(l<$ bandwidth)

17:

foreach Node: $n_{i}$

18:

19: $C(I u) \leftarrow C(I u)+n_{i}(I u)$

20: end for

21: $\boldsymbol{C}(\boldsymbol{M}) \leftarrow H B F(C(I u))$, Filtering content of node $i$

end while

22:

23: $R S S(M) \leftarrow C(M)$ end for

24: $\quad$ end for

25:end

Vehicles are initialized with the wireless parameters and the location of the node. Two random nodes are considered which are in range with one another and the relative acceleration, 
relative velocity and the distance of other vehicles are calculated from those two vehicles. If the distance of the vehicle from the first node (selected vehicle) is smaller than the second node (selected vehicle) and the direction of vehicle is same as the two vehicles along with the velocity and acceleration, then the vehicle lies in the first cluster and vice versa. After clustering the message content is filtered using the Bloom Filters and the probability of true data messages is considered and the routed in the network.

\section{RESULTS AND DISCUSSIONS}

NS-2 is an event based simulator which runs on object oriented $\mathrm{C}++$ at the backend. NS-2 uses OTCL as the frontend where various class objects of $\mathrm{C}++$ are implemented and called. The simulator supports large number of protocols and an animator for the visual output of the network. The proposed methodology is implemented using the NS-2 Simulator with the following parameters shown in table 1. Simulation parameters must be set before the simulation of the environment. The important parameters are channel which is set to wireless channel as the network under consideration is wireless. The model used for the propagation of EM waves is set to be two ray ground. IEEE standard for the MAC, IEEE 802.11 is used for the media access control layer of each vehicle. Antenna must send or receive packets from all the directions so the $\mathrm{C}++$ class of NS2.35, Omni Directional Antenna is used. Number of vehicles is set to be around 100 and simulation time is $300 \mathrm{~s}$.

Table 1 shows the parameters set up for creating the simulation environment.
Table 1: Simulation Parameters

\begin{tabular}{|c|c|}
\hline Channel & Wireless Channel \\
\hline Propagation Model & Two Ray Ground \\
\hline Mac & IEEE 802.11 \\
\hline Antenna & Omni Directional Antenna \\
\hline Number of Vehicles & $300 \mathrm{~s}$ \\
\hline Simulation Time & $200 \mathrm{~m}$ \\
\hline Transmission Range & $10 \sim 40(\mathrm{~m} / \mathrm{s})$ \\
\hline Maximum lane speed & \\
\hline
\end{tabular}

The proposed methodology is implemented using the network simulator ns2.35. The Vehicular Ad hoc Network environment is created and performance of Hybrid Technique in GPSR is compared with the mobility based clustering model for increasing the scalability of the network and the basic GPSR in terms of average Cluster Head $(\mathrm{CH})$ and Cluster Members (CM) duration, their numbers and Routing Overhead, Packet Delivery Ratio and End to End Delay. Figure 1 to 2 shows the average $\mathrm{CH}$ and $\mathrm{CM}$ duration by changing the beacon interval (BI) of the message. With the figure it is clear that there is a little difference between the BI values for both proposed and the mobility based approach. The number of vehicles in the $\mathrm{CH}$ increased with the increase in the velocity while in $\mathrm{CM}$ the number of vehicles decreases. This is because some CMs leaves the cluster and form their own clusters.

0.8

0.7

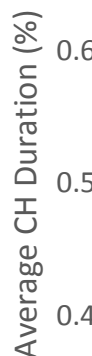

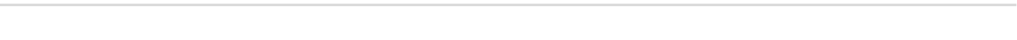

$\mathrm{Bl}=0.5 \mathrm{~s}(\mathrm{MbS}-\mathrm{DC})[17]$

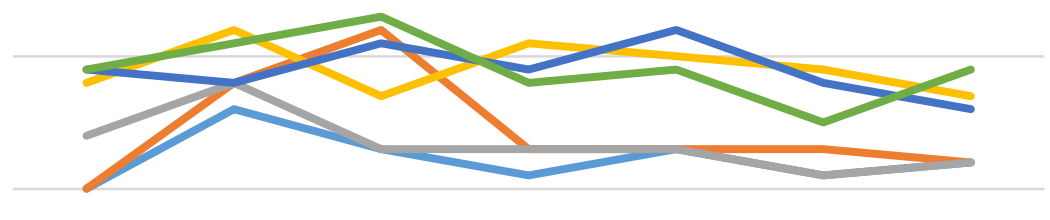

$\mathrm{BI}=1.0 \mathrm{~s}$ (MbS-DC) [17]

$\mathrm{Bl}=2.0 \mathrm{~s}(\mathrm{MbS}-\mathrm{DC})[17]$

$\mathrm{BI}=0.5 \mathrm{~s}(\mathrm{I}-\mathrm{GPSR})$

$\mathrm{BI}=1.0 \mathrm{~s}(\mathrm{I}-\mathrm{GPSR})$

$\mathrm{BI}=2.0 \mathrm{~s}(\mathrm{I}-\mathrm{GPSR})$

0.3

0.2

$\begin{array}{ccccccc}10 & 15 & 20 & 25 & 30 & 35 & 40 \\ & & \text { Maximum Lane Speed (m/s) }\end{array}$

Figure 1:Impact of BI on Average CH Durationplottedbetween Proposed approach and Mbs-DC 
International Journal of Applied Engineering Research ISSN 0973-4562 Volume 12, Number 16 (2017) pp. 5729-5738

(C) Research India Publications. https://dx.doi.org/10.37622/IJAER/12.16.2017.5729-5738

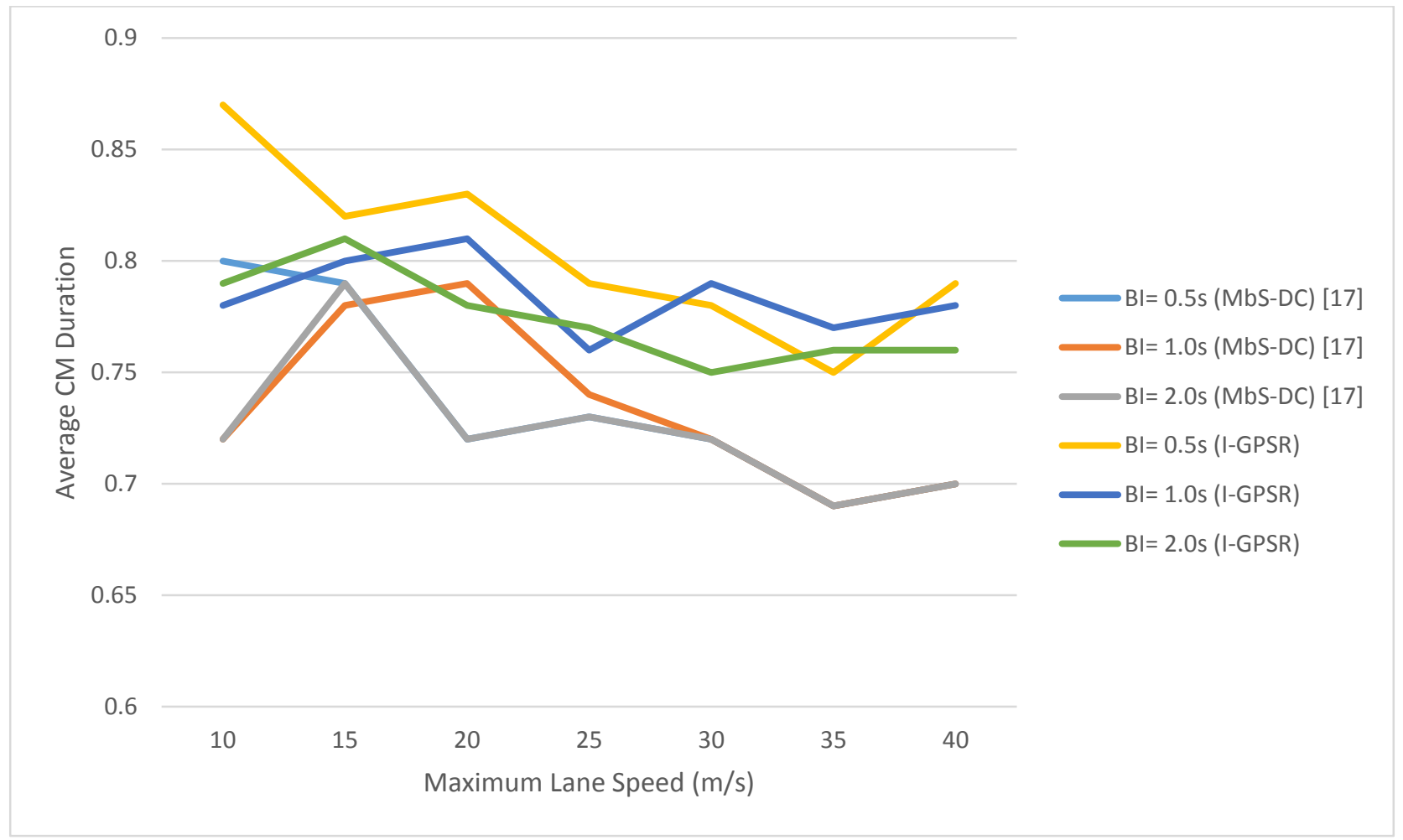

Figure 2:Impact of BI on Average CM Durationplottedbetween Proposed approach and Mbs-DC

Figure 3 to 4 shows the effect on average $\mathrm{CH}$ and $\mathrm{Cm}$ duration with the increase in the lane speed respectively. In the figure it is clear that the average $\mathrm{CH}$ and $\mathrm{CM}$ duration is higher than the mobility based approach because the two Cluster Heads assist the process at any given time simultaneously.

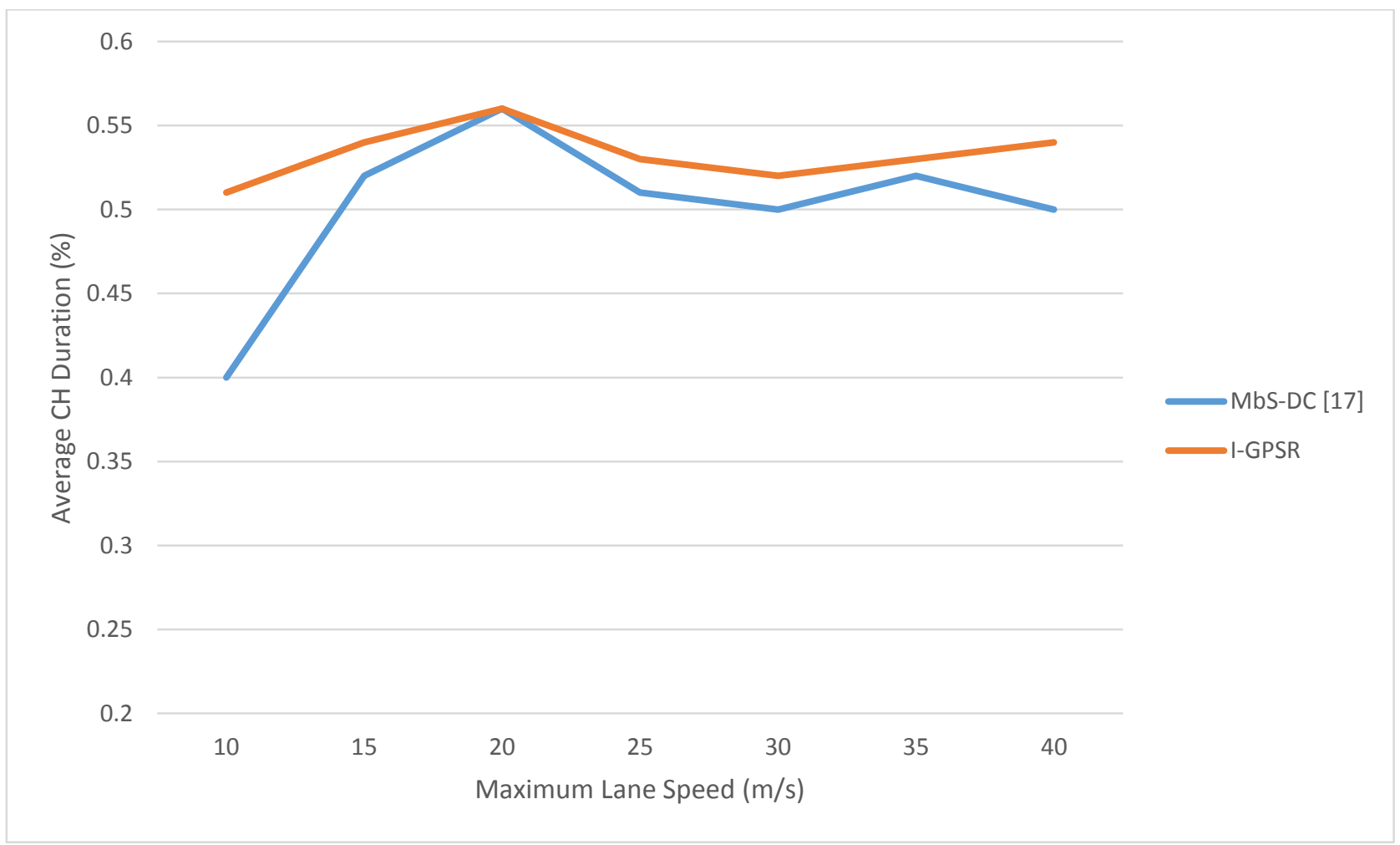

Figure 3: Average $\mathrm{CH}$ duration with the increase in Lane Speedplottedbetween Proposed approach and Mbs-DC 
International Journal of Applied Engineering Research ISSN 0973-4562 Volume 12, Number 16 (2017) pp. 5729-5738

(C) Research India Publications. https://dx.doi.org/10.37622/IJAER/12.16.2017.5729-5738

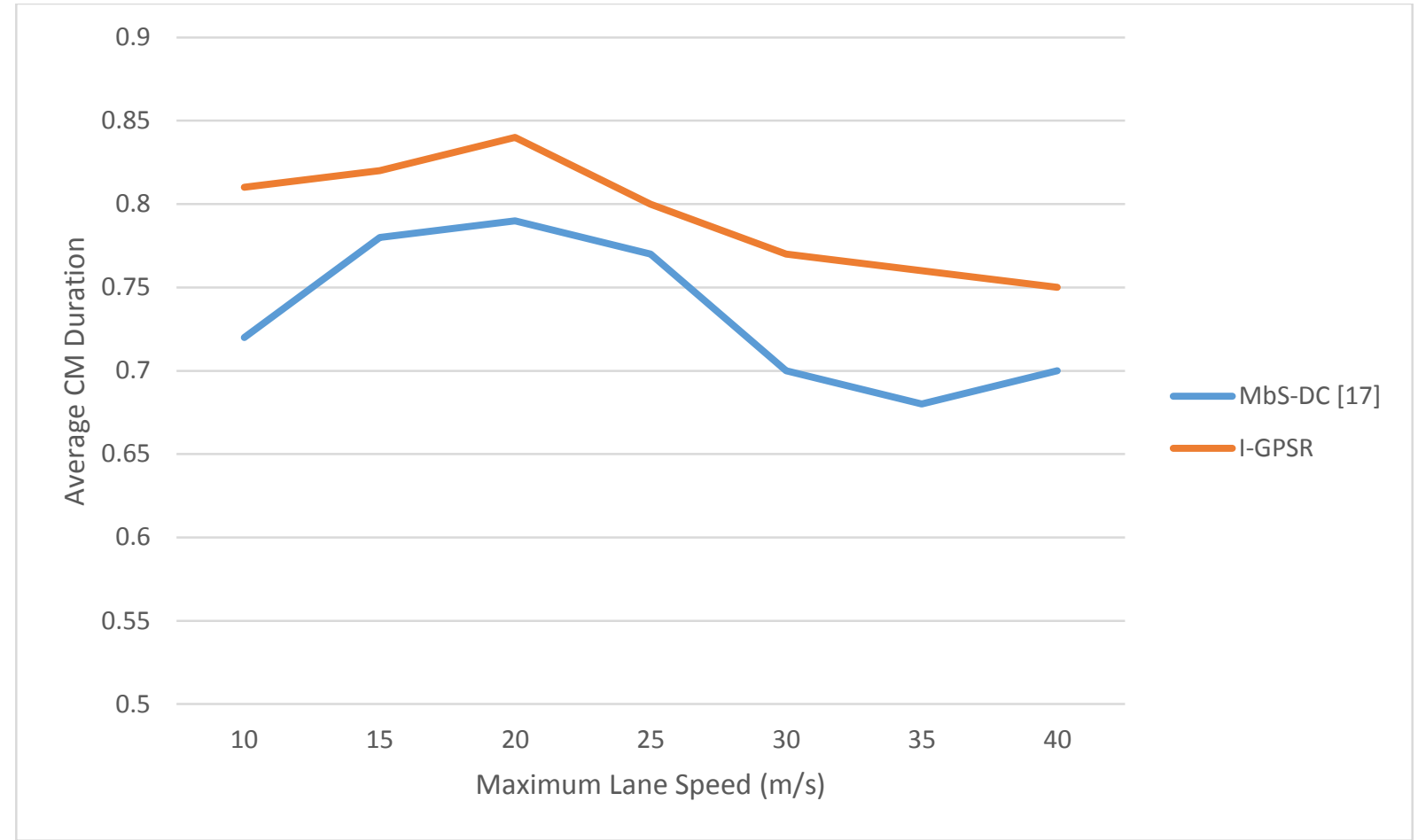

Figure 4: Average CM duration with the increase in Lane Speedplotted between Proposed approach and Mbs-DC

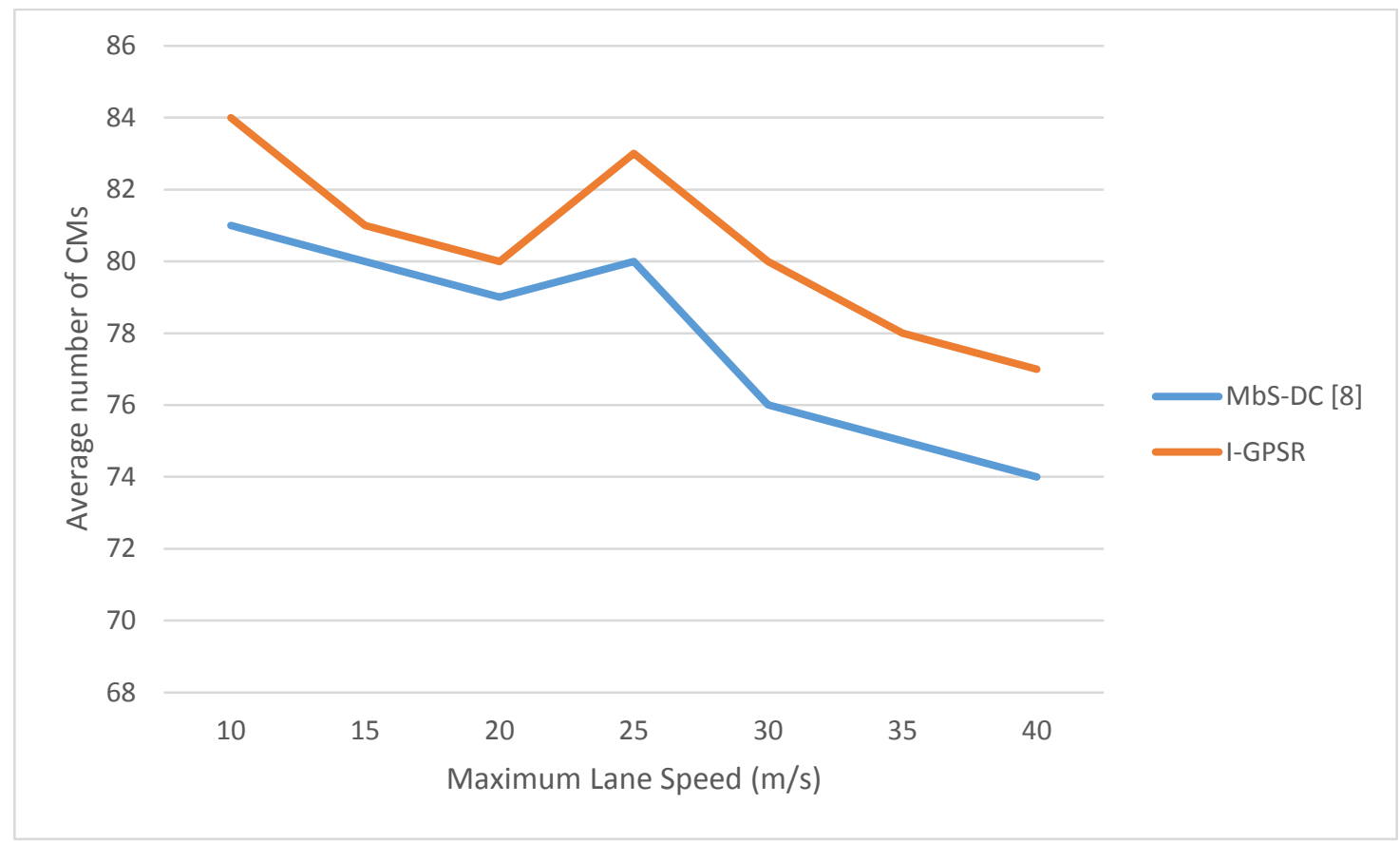

Figure 5: Average number of Cluster Members plotted between Proposed approach and Mbs-DC

Figure 5 shows the number of cluster members in each cluster during the entire process. The figure shows the number of cluster members is higher than that of the mobility based approach. This is due to the fact that the cluster formed considering the acceleration with velocity is more stable as compared to the mobility based approach.
Figure 6 to 8 shows the routing overhead, average end to end delay and packet delivery ratio for the number of vehicles respectively. The proposed approach is compared with theImproved GPSR approach and the results shows an improvement in the parameters because the stability of the clusters increased and the bandwidth is effectively utilized and the number of packets lost in the network decreases. 
International Journal of Applied Engineering Research ISSN 0973-4562 Volume 12, Number 16 (2017) pp. 5729-5738 (C) Research India Publications. https://dx.doi.org/10.37622/IJAER/12.16.2017.5729-5738

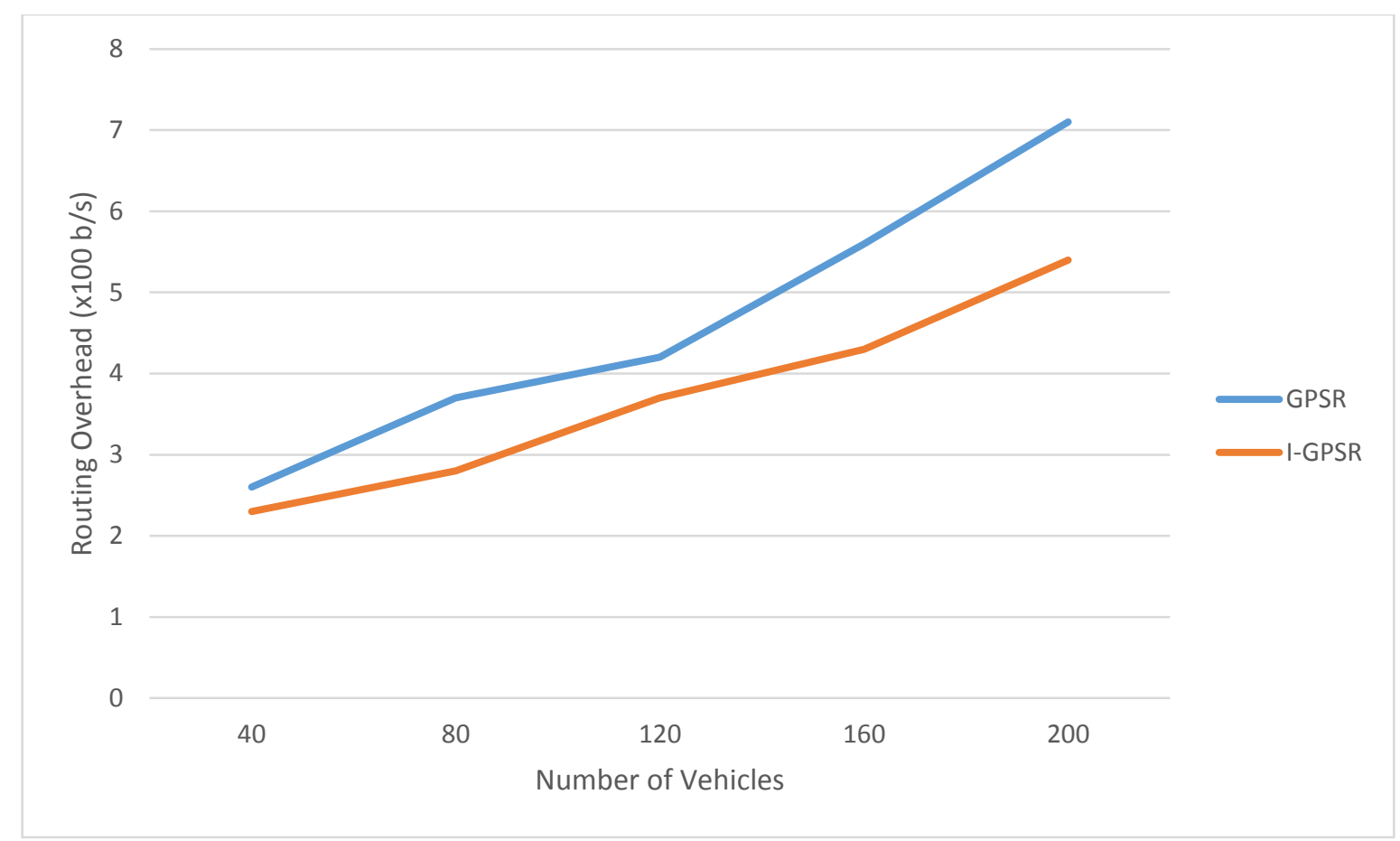

Figure 6: Routing Overhead Vs Number of nodes

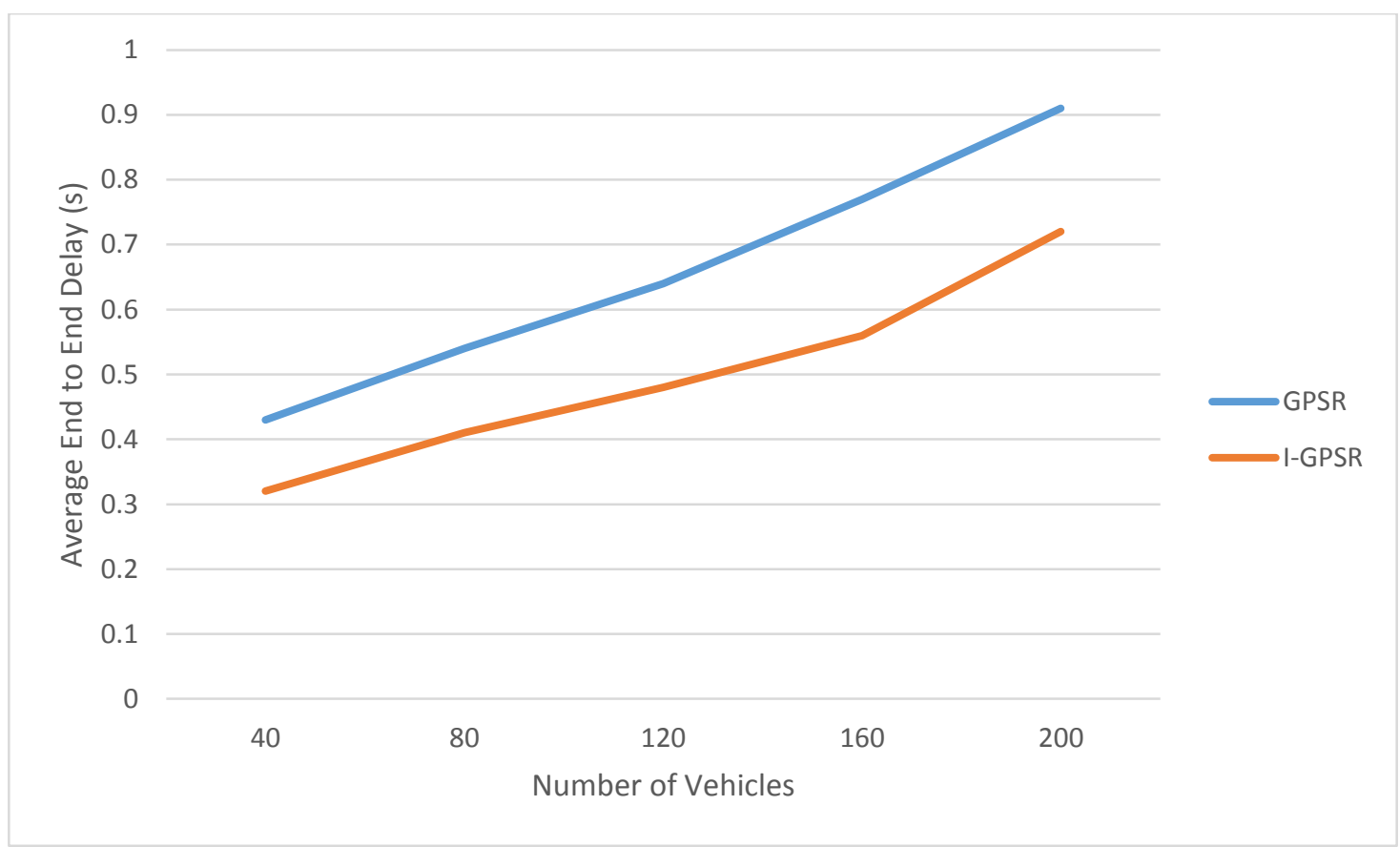

Figure 7: Average End to End Delay Vs Number of nodes 
International Journal of Applied Engineering Research ISSN 0973-4562 Volume 12, Number 16 (2017) pp. 5729-5738

(C) Research India Publications. https://dx.doi.org/10.37622/IJAER/12.16.2017.5729-5738

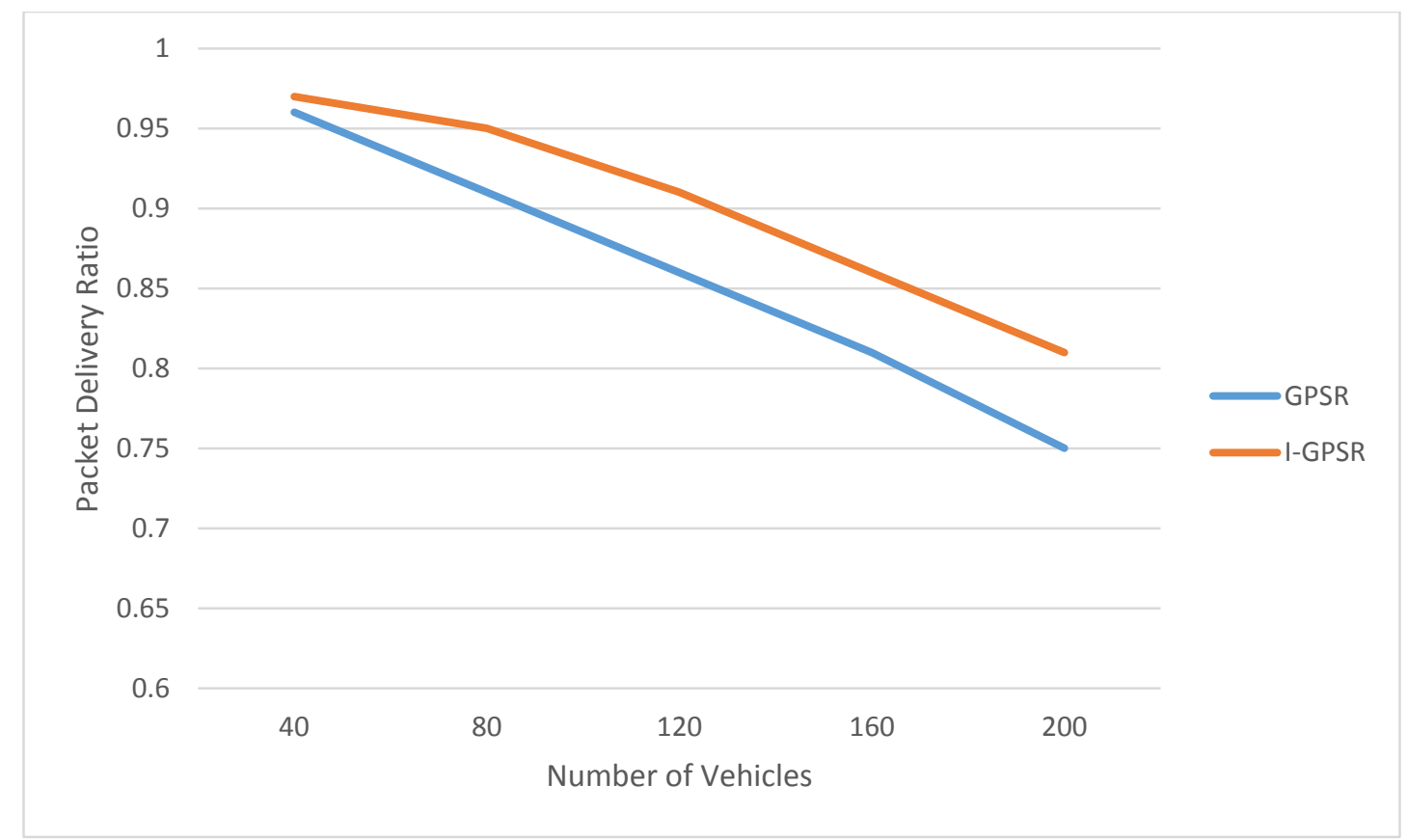

Figure 8: Packet Delivery Ratio Vs Number of nodes

The proposed algorithm is then compared with [25] varying the number of nodes in the network. Figure 9-10 shows that the proposed approach performs better in comparison to the approaches in [25]. The reason for the increase in Cluster Head $(\mathrm{CH})$ and Cluster Member $(\mathrm{CM})$ duration is the clustering approach used is stable because of the consideration of velocity, acceleration and direction. Because of the above consideration only those nodes join the network which satisfies these conditions and remain likely the same for a longer duration.

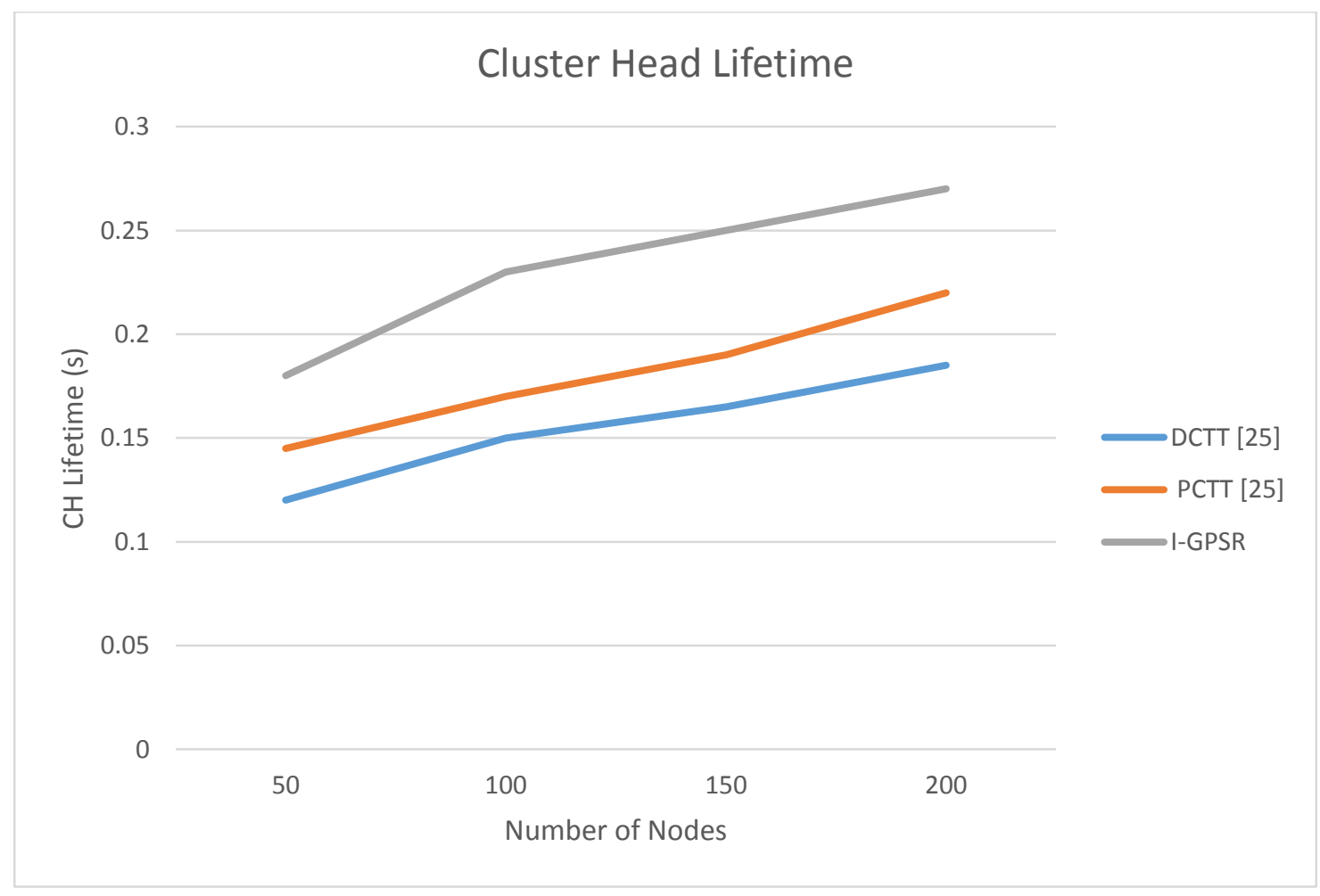

Figure 9: Variation of Cluster Head Lifetime with Number of Nodes 


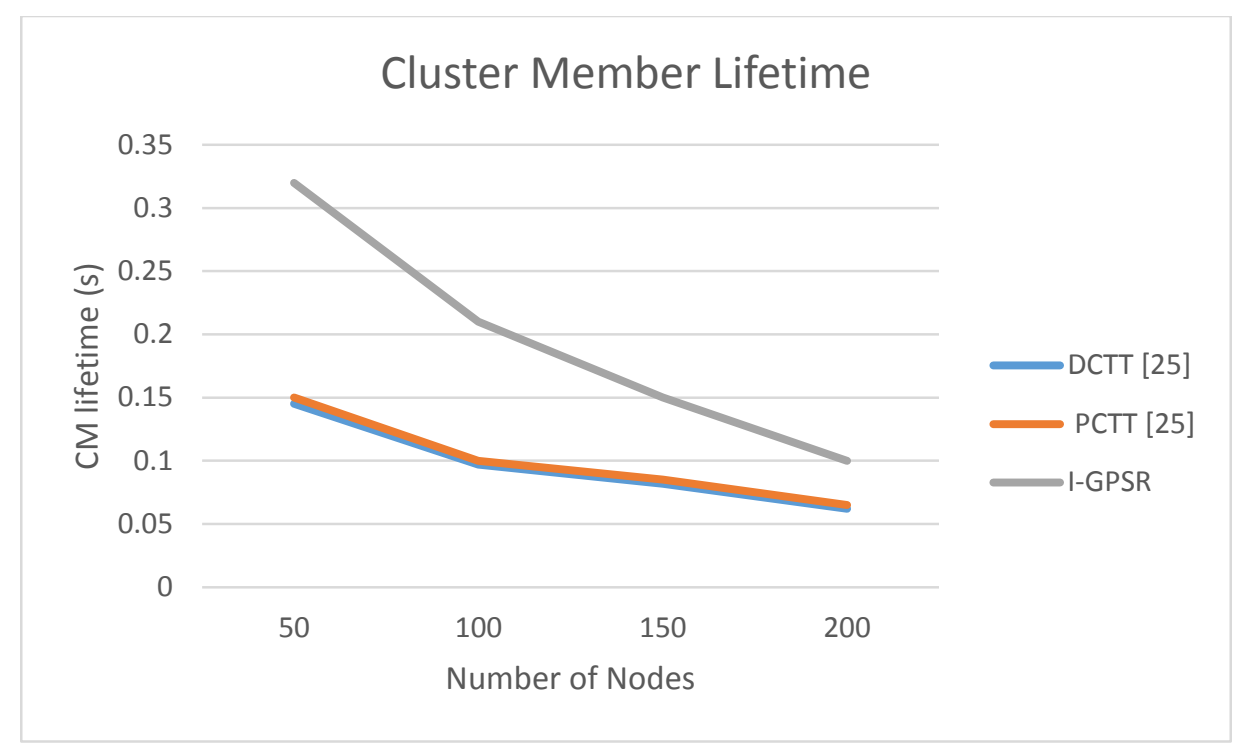

Figure 10: Variation of Cluster Member Lifetime with Number of nodes

The Clustering Control overhead shown in figure 11 is less as compared to the approaches given in [25]. This is due to the fact that the cluster is not flooded with the control messages by the $\mathrm{CH}$. Also the filtering scheme is used which filters the messages from the cluster members before sending it to the
RSS. As the cluster members increases in the network, the number of cluster control and data messages also increased and are managed using the time division scheme at the cluster head side. The new messages can be accepted and handled once the filtering operation on the $\mathrm{CH}$ side is performed.

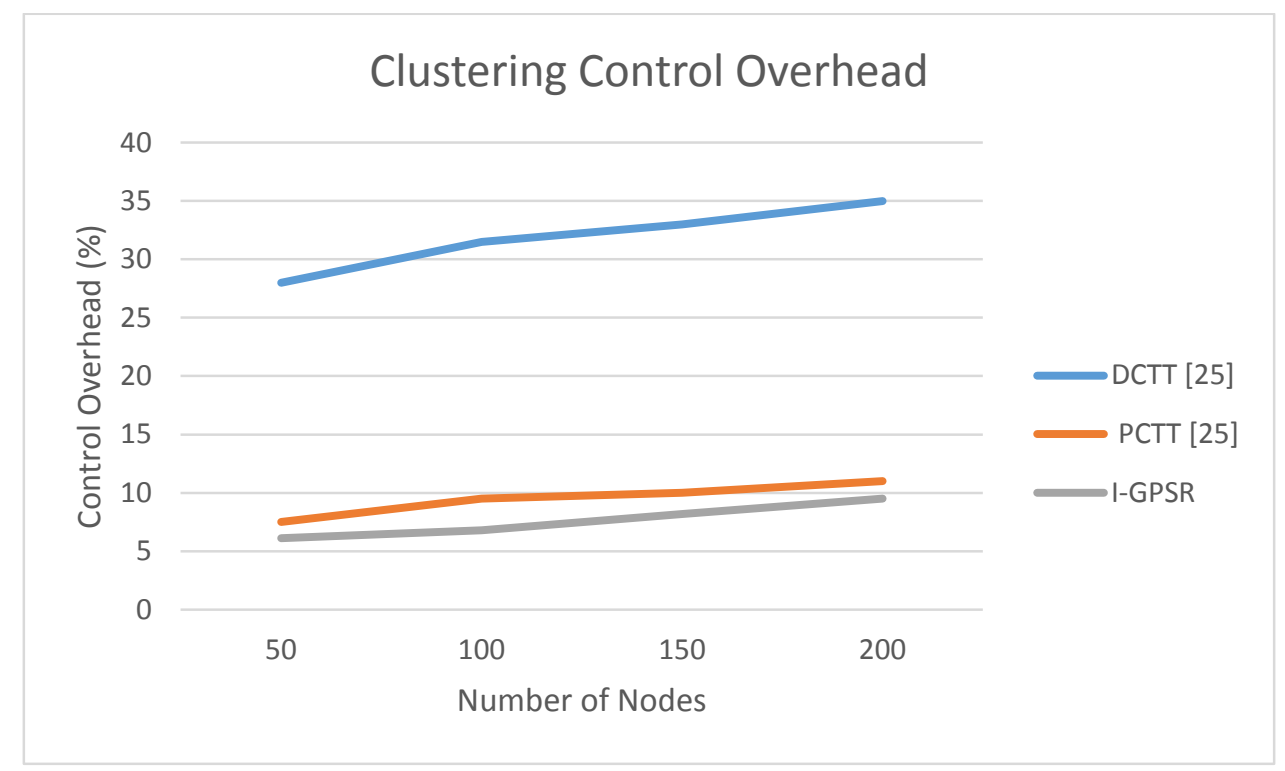

Figure 11: Variation of Control Overhead with Number of nodes

\section{CONCLUSION}

With the growing size of the network, network scalability and adaptability of the new vehicles in the current ecosystem is widely researched area. The increase in the vehicles with scalable solution of the network considering the performance parameters to not degrade is an important challenge and many techniques have been suggested in recent years for the same. The present work tries to compare the improved hybrid GPSR with the mobility based clustering in VANET and GPSR technique. Hybrid GPSR is a combination of the clustering technique and the Content filtering technique. The clustering in the network is based on the location parameters matrix technique and the filtering is based on the bloom filtering technique. A VANET environment is created based on the parameters given in the table. For the same environments, the hybrid GPSR approach outperforms the basic approaches. The proposed approach is also compared with the clustering based tracking algorithms for the improvement in scalability by varying the number of vehicles in the network. In future different clustering techniques must be used with the content based technique to form hybrid approaches and compared with the existing approaches. 
International Journal of Applied Engineering Research ISSN 0973-4562 Volume 12, Number 16 (2017) pp. 5729-5738

(C) Research India Publications. https://dx.doi.org/10.37622/IJAER/12.16.2017.5729-5738

\section{REFERENCES}

[1] Alazzawi, Lubna K., Ali M. Elkateeb, Aiyappa Ramesh, and WaleedAljuhar. "Scalability analysis for wireless sensor networks routing protocols." InAdvanced Information Networking and Applications-Workshops, 2008. AINAW 2008. 22nd International Conference on, pp. 139-144. IEEE, 2008.

[2] Shen, Haiying, and Lianyu Zhao. "ALERT: An anonymous location-based efficient routing protocol in MANETs." Mobile Computing, IEEE Transactions on 12, no. 6 (2013): 1079-1093.

[3] Hong, Xiaoyan, KaixinXu, and Mario Gerla. "Scalable routing protocols for mobile ad hoc networks." IEEE network 16, no. 4 (2002): 11-21.

[4] Mikki, Mohammad A. "Energy efficient location aided routing protocol for wireless MANETs." arXiv preprint arXiv:0909.0093 (2009).

[5] Tee, C. A. T. H., and A. Lee. "Adaptive reactive routing for VANET in city environments." In Pervasive Systems, Algorithms, and Networks (ISPAN), 2009 10th International Symposium on, pp. 610-614. IEEE, 2009.

[6] Phoummavong, Phonepadith, Keisuke Utsu, Chee Onn Chow, and Hiroshi Ishii. "Location-aided route discovery mechanism based on two-hop neighbor information for ad hoc network." The Journal of Supercomputing 72, no. 3 (2016): 1201-1214.

[7] Hannes Hartenstein, Jing Tian, HolgerFussler, Dagmar Hermann, and Martin Mauve. "A routing strategy for vehicular ad hoc networks in city environments." In Intelligent Vehicles Symposium, 2003.Proceedings. IEEE, pp. 156-161. IEEE, 2003.

[8] Ren, Mengying, et al. "A mobility-based scheme for dynamic clustering in vehicular ad-hoc networks (VANETs)." Vehicular Communications (2016).

[8] Yu, Yu-Ting, Mario Gerla, and M. Y. Sanadidi. "Scalable VANET content routing using hierarchical bloom filters." Wireless Communications and Mobile Computing 15, no. 6 (2015): 1001-1014.

[9] Rajput, Ubaidullah, Fizza Abbas, HasooEun, and Heekuck Oh. "A Hybrid Approach for Efficient Privacy Preserving Authentication in VANET." IEEE Access (2017).

[10] Wang, Yu, Jun Zheng, and Nathalie Mitton. "Delivery Delay Analysis for Roadside Unit Deployment in Vehicular Ad Hoc Networks with Intermittent Connectivity." (2016).

[11] Mishra, Yogesh, Ashish Singhadia, and Rashmi Pandey. "Energy Level Based Stable Election Protocol in Wireless Sensor Network." International Journal of Emerging Trends and Technology 17, no. 1 (2014): 3238.

[12] Deniz, Fatih, HakkiBagci, Ibrahim Korpeoglu, and Adnan Yazicı. "An adaptive, energy-aware and distributed fault-tolerant topology-control algorithm for heterogeneous wireless sensor networks." Ad Hoc Networks 44 (2016): 104-117.

[13] DuanCuiqin,SunJingjing,ZhouDuan,ZhangJianxian. "An energy efficient regional partitioned clustering routing algorithm for wireless sensor networks" .In:Second international conference on intelligent networks and intelligent systems(ICINIS-
2009).IEEE;2009.p.205-8.

[14] Wang. C, Sohraby. K., Li.B, Daneshmand. M, Hu.Y, "A Survey of transport protocols for wireless Sensor Networks", IEEE, 2006.

[15] Mansouri. V.S, Afsari. B, Shahmansouri. H, " A simple Transport protocol for wireless sensor Networks", IEEE, 2005.

[16] Wang, Sheng-Shih, and Ze-Ping Chen. "LCM: a linkaware clustering mechanism for energy-efficient routing in wireless sensor networks." Sensors Journal, IEEE 13.2 (2013): 728-736.

[17] Malhi, AvleenKaur, and ShaliniBatra. "Fuzzy-based trust prediction for effective coordination in vehicular ad hoc networks." International Journal of Communication Systems (2016).

[18] Ao, Buke, Yongcai Wang, Lu Yu, Richard R. Brooks, and S. S. Iyengar. "On Precision Bound of Distributed Fault-Tolerant Sensor Fusion Algorithms."ACM Computing Surveys (CSUR) 49, no. 1 (2016): 5.

[19] Liu, Xing, Haiying Zhou, ShengwuXiong, Kun Mean Hou, Christophe De Vaulx, and Hongling Shi. "Research of a resource-efficient, real-time and faulttolerant wireless sensor network system." Journal of Information Security and Applications (2016).

[20] Wang, Neng-Chung, Jong-Shin Chen, Yung-Fa Huang, and Si-Ming Wang. "A Greedy Location-Aided Routing Protocol for Mobile Ad Hoc Networks."InWSEAS International Conference.Proceedings. Mathematics and Computers in Science and Engineering, edited by

[21] Muñoz, Alberto Gordillo. "Multicast over Vehicle Ad Hoc Networks."

[22] David B. Johnson Saha, Amit Kumar. "Modeling mobility for vehicular ad-hoc networks." In Proceedings of the 1st ACM international workshop on Vehicular ad hoc networks, pp. 91-92. ACM, 2004.

[23] Lochert, Christian, Hannes Hartenstein, Jing Tian, HolgerFussler, Dagmar Hermann, and Martin Mauve. "A routing strategy for vehicular ad hoc networks in city environments." In Intelligent Vehicles Symposium, 2003.Proceedings. IEEE, pp. 156-161. IEEE, 2003.

[24] Toutouh, Jamal, and Enrique Alba. "Light commodity devices for building vehicular ad hoc networks: An experimental study." Ad Hoc Networks 37 (2016): 499511.

[25] Khakpour, Sanaz, Richard W. Pazzi, and Khalil ElKhatib. "Using clustering for target tracking in vehicular ad hoc Networks."Vehicular Communications. 9(2017):83-96.

[26] H. Takagi, L. K. (1984). Optimal transmission ranges for randomly distributed packet radio terminals. IEEE Transactions on Communications, vol. 32, no. 3, (pp. 246-257). 